\title{
Electron diffraction analysis of individual single-walled carbon nanotubes
}

\author{
Jannik C. Meyer, ${ }^{1, \text { f }}$ Matthieu Paillet, ${ }^{2}$ Georg S. Duesberg, ${ }^{3}$ and Siegmar Roth ${ }^{1}$ \\ ${ }^{I}$ Max Planck Institute for Solid State Research, Stuttgart, Germany \\ ${ }^{2}$ Laboratiore des Colloides, Verres et Nanomateriaux, Universite de Montpellier II, France \\ ${ }^{3}$ Infineon Technologies Corporate Research, Munich, Germany
}

\begin{abstract}
We present a detailed electron diffraction study of individual single-walled carbon nanotubes. A novel sample preparation procedure provides well-separated, long and straight individual single-shell nanotubes. Diffraction experiments are carried out at $60 \mathrm{kV}$, below the threshold for knock-on damage in carbon nanotubes. We describe experimental parameters that allow single-tube electron diffraction experiments with widely available thermal emission transmission electron microscopes. Further, we review the simulation of diffraction patterns for these objects.
\end{abstract}

PACS numbers: 61.14.Lj, 61.46.+w, 81.07.-b, 81.07.De

Keywords: Carbon nanotubes, Electron diffraction, Nanotube and TEM sample preparation,

\section{INTRODUCTION}

From a diffraction analysis, it is possible to derive the exact lattice structure of an individual single-walled carbon nanotube (SWNT). The nanotube sections used for electron diffraction experiments in a transmission electron microscope (TEM) contain only a small number of identical atoms that interact weakly with the electron beam. Therefore the diffraction patterns can be easily understood and simulated by simple approximations. For the same reasons, obtaining a diffraction pattern is an experimental challenge. Both the simulation of diffraction patterns, and diffraction experiments at acceleration voltages below the knock-on damage threshold are described. The latter is important for non-destructive determination of the nanotube structure, required for electron diffraction experiments in combination with other single-tube measurements on the same nanotube.

Detailed diffraction studies exist on multi-walled [1, 2, 3, 4] and double-walled [5, 6, 7, 8] carbon nanotubes, and bundles of single-walled carbon nanotubes $[9,10,11,12,13,14,15$, 16, 17, 18, 19]. Diffraction experiments on individual SWNTs are an experimental challenge because they require finding sufficiently long, straight sections of an individual nanotubes (raw material is mostly bundled) that are stable throughout the exposure. Nanotubes with more than one shell are usually stiff enough to exhibit long, straight sections with standard sample preparation procedures. SWNTs, on the other hand, tend to curve, and to combine into bundles. A curved nanotube is not a periodic one-dimensional structure. Few examples of sharp diffraction patterns from individual SWNTs exist in the literature [20, 21, 22]. However with the sample preparation procedure shown in this work, long straight sections of individual SWNTs are easily obtained. We have developed a way to suspend nanotubes in a metal grid that provides long and straight tube sections. In the resulting samples, tubes suitable for diffraction are easily found and reliably produce a diffraction pattern with a close to normal incidence. With the given

\footnotetext{
*Electronic address: j.meyer@ fkf.mpg.de
}

conditions, more than $50 \%$ of the diffraction exposures result in a pattern that can be uniquely assigned to a nanotube structure. The "failed" exposures are attributed to curved or strongly vibrating tubes, or to objects other than the tube illuminated by the beam. Indices for more than 50 nanotubes were determined so far. It is a reliable procedure, which is a prerequisite for the combination with other experiments on the same tube. We have shown the combination of TEM imaging and transport measurements previously [23], and plan to extend this method towards transport and electron diffraction on the same molecule.

For the simulated diffraction images, a computer program was written which produces the nanotube structure for given indices, and then calculates the diffraction intensities using the principles and equations shown in the simulations section. We determine the nanotube structure by comparison with simulated images, and find that there is exactly one nanotube structure which matches the experimental pattern. For an analytical analysis of the diffraction patterns based on the helical structure, see e.g. [3, 12, 24, 25, 26]. We note that the index determination solely from relative peak distances as described in [27, 28] is valid only for precisely normal incidence, a condition that is not easily established experimentally. The comparison with simulations, in contrast, allows to determine the indices and at the same time to measure the incidence angle by varying these parameters until the simulated pattern matches the experimental one.

Other techniques for identifying the structural indices are atomic-resolution TEM imaging [29, 30, 31], Raman spectroscopy [32, 33], fluorescence spectroscopy [34, 35, 36], and scanning tunnelling microscopy and spectroscopy (STM/STS) [37, 38, 39, 40]. Electron diffraction and high-resolution images can be directly related to a pair of carbon nanotube structural indices $(\mathrm{n}, \mathrm{m})$, while spectroscopic techniques rely on a modelization of electronic and vibrational properties of carbon nanotubes. A quantitiative analysis of the index distribution is complicated by a different response for different nanotubes both in Raman [41] and fluorescence spectroscopy. Further, fluorescence spectroscopy detects only semiconducting nanotubes. 


\section{SAMPLE PREPARATION}

Carbon nanotubes are grown by chemical vapour deposition (CVD) on highly doped Si substrates with a 200nm oxide layer. Different CVD growth conditions were used, as described in [42, 43, 44]. A grid structure is prepared on top of the nanotubes by electron beam lithography, thermal evaporation of $3 \mathrm{~nm} \mathrm{Cr}$ and $110 \mathrm{~nm} \mathrm{Au}$, and a lift-off process. The sample is cleaved so that the structure is on the cleaved edge of the substrate (Fig. 11). There are two possible etching processes to obtain the free-standing structure (Fig. 1b):
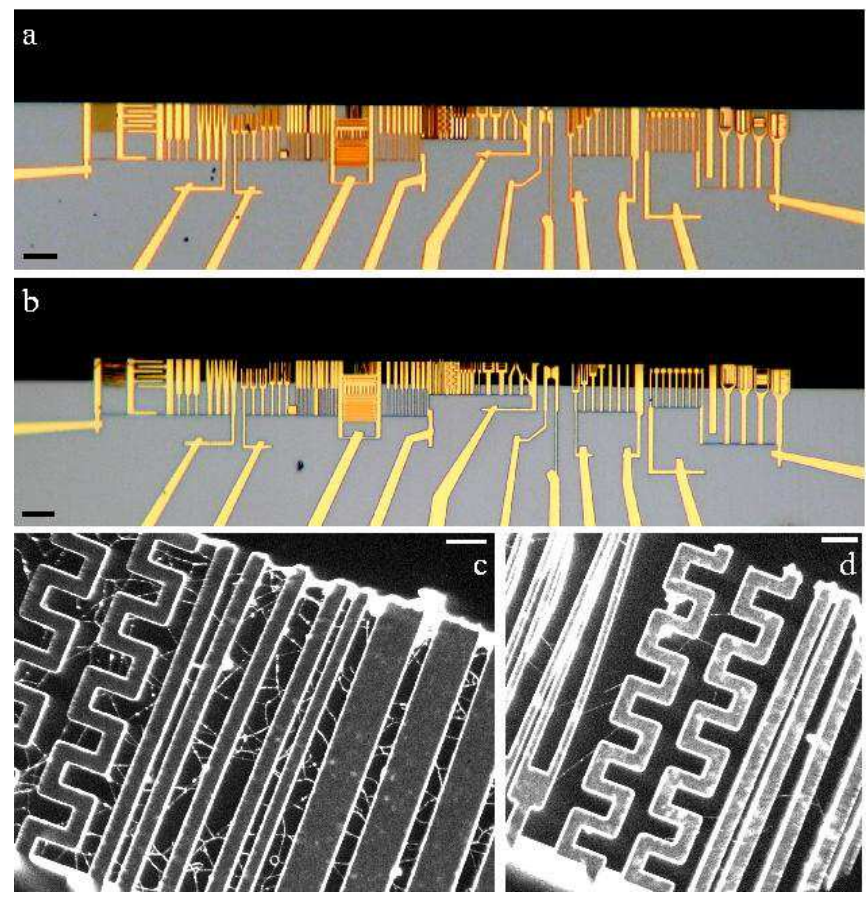

Figure 1: Sample preparation. (a) The grid structure, prepared by electron beam lithography on top of the nanotube network, close to the cleaved edge of the substrate. (b) After etching, part of the grid is free-standing. This free-standing part can be observed by TEM. (c) shows a dark-field mode TEM image of a high-density sample, (d) a low-density sample. Especially in lower-density samples, long straight individual SWNTs of high purity (depending on CVD conditions) are obtained. The dark-field mode is used here since the clean SWNTs are not seen in bright-field mode at lower magnifications. Scale bars are $10 \mu \mathrm{m}$ in (a) and (b), and $1 \mu \mathrm{m}$ in (c) and (d).

1. The more simple process is a 6 hours etch in $30 \% \mathrm{KOH}$ at $60^{\circ} \mathrm{C}$. The $\mathrm{KOH}$ removes the bulk $\mathrm{Si}$, and slowly the oxide layer. Thus, the structure is undercut mostly from the side. The etching process has to be stopped when the oxide layer is completely removed in the freestanding part, but still present on the substrate. The etch rate of the bulk Si can be controlled by electrically biasing the substrate with respect to the etching solution. The etch process is monitored with an optical microscope, and the bias is switched to a positive potential on the bulk $\mathrm{Si}$ as soon as the free-standing part has the desired width. This stops the etching on the bulk Si, while the etching on the oxide layer continues.

2. A two-step etch process consists of a TMAH etch ( $15 \%$, $60^{\circ} \mathrm{C}$ ) to underetch the structure and oxide layer from the side of the cleaved edge, followed by a buffered $\mathrm{HF}$ etch $(6.5 \%, 2 \mathrm{~min})$ to remove the oxide layer. The width of the free-standing part can be controlled by the time of the TMAH etch (typically 1-3 hours). This process allows the preparation of more "fragile" structures, like large free-standing patterns, very long suspended nanotubes, or lithographically defined metal objects suspended on carbon nanotubes. The TMAH etch step is monitored with an optical microscope, and stopped when the free-standing part has reached a sufficient width.

Both processes are followed by transferring the sample into water, then into isopropanol, and finally into acetone (VLSI grade). A specially designed sample holder prevents the edge of the sample from drying. Finally, a critical point drying step with carbon dioxide is carried out. The substrates are small enough to be glued into conventional TEM rings. The etching process does not damage the nanotubes: The diffraction patterns show a well preserved crystallinity, and the Raman spectra [45] show no unusual features and a low D line.

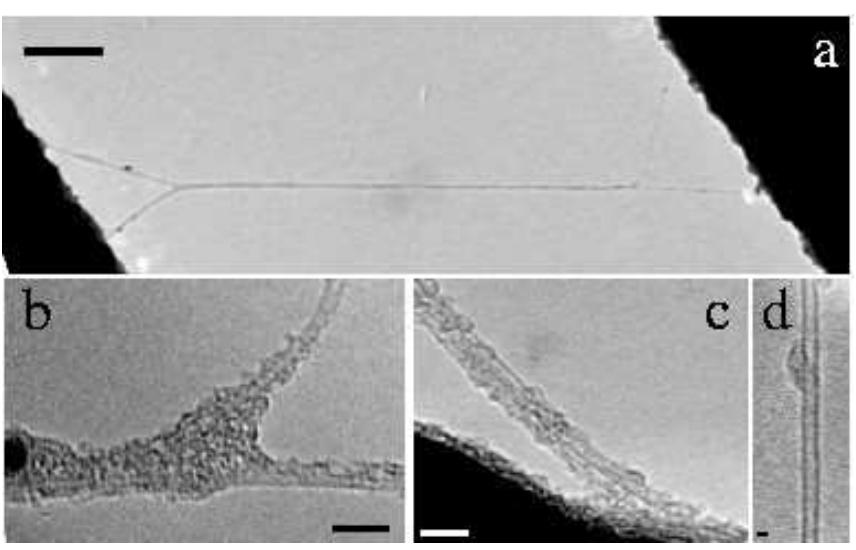

Figure 2: (a) Stretching of a small bundle by coalescence of the two nanotubes. We often observe small bundles splitting near the contact structure. These nanotubes were most likely separated before the etching process, but close together. They are fixed at the contacts, but the central part is free to move after etching. Due to van der Waals attraction the central sections have formed a bundle. As a result, the tube sections are straightened. (b) is a close-up on the right splitting point in (a). (c) shows a nanotube end (a bundle in this case) close to the contact. Tubes often appear curved just at the ends, suggesting that they are stretched by an attractive force towards the contacts. Most of the amorphous carbon is deposited during the TEM analysis: (d) shows a nanotube section after quickly zooming in to a high magnification. Scale bars are $100 \mathrm{~nm}(\mathrm{a}), 5 \mathrm{~nm}(\mathrm{~b}+\mathrm{c})$ and $1 \mathrm{~nm}(\mathrm{~d})$.

As a result of this procedure, we obtain well-defined nanotube samples with nanotubes nearly orthogonal to the beam. In low-density samples (Fig. 11), approximately $50 \%$ of the nanotubes are individual and well stretched between the 
contacts for a diffraction analysis. In higher-density samples (Fig. 11), bundling occurs more frequently, and curved free-standing bundles appear. Individual tubes appear to be stretched by van der Waals attraction towards the contacts. Especially if they are not ending orthogonal to the contact, they curve towards a parallel alignment with the contact edge. Similarly, stretching is observed in small bundles (which are most likely formed after etching, when the tubes are free to move). An example is shown in Fig. 2

We want to point out that carbon nanotubes, grown by CVD directly on a bulk substrate, can not usually be investigated by TEM and electron diffraction. They become accessible only due to our sample preparation procedure. This permits a quality control of CVD grown nanotubes, and provides information not available from AFM or SEM investigations about bundling, precise diameters, number of shells, and amorphous carbon coating from TEM, and index distribution from diffraction analysis.

Instead of CVD grown nanotubes, it is also possible to use nanotubes deposited on the substrates from a suspension. However we find that this leads to a high amount of SWNT bundles.

\section{SIMULATIONS}

Two different ways to calculate the diffraction pattern of a SWNT are used. The real-space path summation approach is computationally expensive but easy to understand and implement. It produces the correct peak positions, but not the right intensities. Our determination of the nanotube structure only depends on the peak positions. The alternative is computing the Fourier transform of the projected atomic potentials. This is the standard approach for thin objects that do not require multi-slice algorithms. However, peak positions deviate slightly from the correct values because the curvature of the Ewald sphere is not taken into account.

Individual single-walled carbon nanotubes are one of the few systems of which the interaction with electrons in a TEM is well described by the weak phase object approximation (WPOA) and the 1st Born approximation. We use nonrelativistic quantum mechanics with relativistically corrected values for the electron mass $m$ and wavelength $k$. It has been shown that electron microscopic problems are well described in this way [46, 47, 48].

\section{A. Path summation approach}

Due to the small number of atoms in our one-dimensional samples it is possible to numerically calculate diffraction images from basic physical principles in a real-space representation. We consider an electron propagating from $\left(r_{0}, t_{0}\right)$ to $\left(r_{1}, t_{1}\right)$. In the most general case, the complex probability amplitude $\Psi\left(r_{1}, t_{1}\right)$ is given by a summation over all paths starting from $\Psi\left(r_{0}, t_{0}\right)$ [49, 50]:

$$
\Psi\left(r_{1}, t_{1}\right)=\Psi\left(r_{0}, t_{0}\right) \cdot \sum_{\text {paths }} e^{\frac{i}{\hbar} S}
$$

where $S$ is the action along the path. For propagation in free space, the summation can be replaced the term for the classical path, multiplied with a constant $c_{1}^{\prime}$ or $c_{1}$ [50]:

$$
\sum_{\text {paths }} e^{\frac{i}{\hbar} S}=c_{1}^{\prime} \cdot e^{\frac{i}{\hbar} S_{c l} .}=c_{1} \cdot \frac{1}{|r|} e^{i(k r-\omega t)}
$$

If we idealize the atoms in an object as point scatterers, we can divide the summation into the scattering events and piecewise propagation in free space. This is illustrated in Figure 3 We consider the propagation from the source to the detector as the sum of the direct contribution, plus the paths including a single scattering event, two events and so on. The sum is thus written as:

$$
\begin{aligned}
\sum_{\text {paths }} e^{\frac{i}{\hbar} S} & =c_{1} \cdot \frac{1}{\left|r_{S D}\right|} e^{i k r_{S D}} \\
& +c_{1}^{2} \cdot \sum_{n} \frac{1}{\left|r_{S A_{n}}\right|\left|r_{A_{n} D}\right|} e^{i k r_{S A_{n}}} f_{n} e^{i k r_{A_{n} D}} \\
& +c_{1}^{3} \cdot \sum_{n} \sum_{m} \ldots
\end{aligned}
$$

Here, $r_{S D}$ is the distance from the source to the detector, $r_{S A_{n}}$ the distance from the source to atom $n$, and $r_{A_{n} D}$ the distance from atom $n$ to the detector. The summations are over all atoms of the object, $f_{n}$ is the factor describing the scattering event at atom $n$. For a set of identical atoms, the actual value of the scattering factor $f_{n}$ is not important. The above summation represents Huygens principle of a spherical wave originating from every atom in the sample. Considering only the 0 th and 1 st order contributions, i.e. lines 1 and 2 in the above summation, is the equivalent of the 1st Born approximation for atoms idealized as point scatterers.

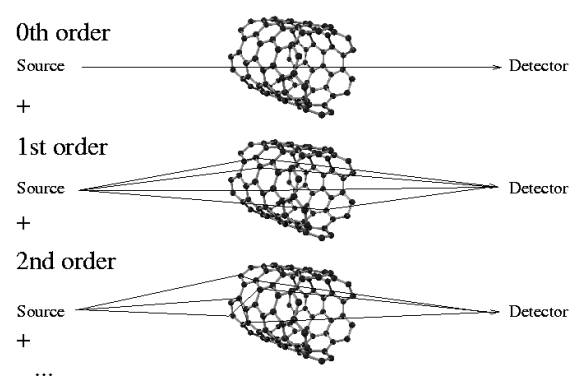

Figure 3: Contributions to the path summation, sorted by the number of interactions. The phases $e^{i k r}$ along each path are summed up. It is equivalent to the 1 st Born approximation to consider only 0th and 1 st order contributions. The summation is carried out for every point in the simulated image. 
For objects containing a small number of atoms, the above summation can be carried out numerically. Given the small number of light (i.e., weakly scattering) atoms in an individual single-walled carbon nanotube, the 1 st order approximation is reasonable. Assuming a detector at a large distance from the nanotube, diffraction patterns can be calculated numerically directly from (3). Although reciprocal-space approaches for image simulation (shown below) are more efficient in terms of required computing time, the real-space summation can conveniently be carried out on a standard PC for the small number of atoms in a carbon nanotube. The length of the simulated nanotube section determines the width of the peaks. A $50 \mathrm{~nm}$ long simulated nanotube is sufficient for calculating a highquality diffraction image.

\section{B. Fourier space approach}

Alternatively, diffraction patterns of carbon nanotubes can be calculated from single-slice projected potentials. This is a widely used method for sufficiently thin objects that do not require multi-slice algorithms. Detailed descriptions are found in $[48,51,52,53,54]$. Here we shortly summarize the approximations and calculations we use.

Within the 1st Born approximation the diffraction pattern can be obtained from a Fourier transform of the scattering potential $V(r)$. In the case of small momentum transfer, we can approximate the observed section of the Ewald sphere by a planar section. In this approximation the diffraction pattern is the 2D-Fourier transform of the projected scattering potential.

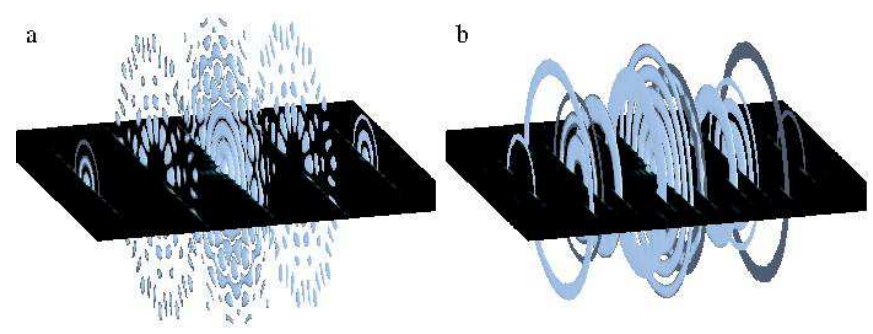

Figure 4: Three-dimensional Fourier transformation of a $(7,7)$ nanotube (a) and an $(8,3)$ nanotube (b). An isosurface is drawn around the higher intensity volume elements, shown in a perspective view. For a bulk crystal, this image would show the reciprocal lattice points. But since we have a one-dimensional structure, we obtain features that are extended in two dimensions: The diffraction intensities are cumulated on discs orthogonal to the tube axis. The planar section corresponds to the section of the Ewald sphere that would be observed in an electron diffraction experiment with normal incidence on the tube. For the $(7,7)$ nanotube, a discrete 14 -fold rotational symmetry around the tube axis is present in the Fourier transform.

In Figure 4 the three-dimensional Fourier transform of a carbon nanotube is calculated, and a planar cut corresponding to an observable section of the Ewald sphere is shown. Since the nanotube is a periodic one-dimensional structure, the intensity in reciprocal space is cumulated on discs orthogonal to the tube axis. The spacing of these discs corresponds to the periodicities along the tube axis, while the radial intensity distribution is determined by the tube diameter. The armchair and zigzag nanotubes exhibit a discrete rotational symmetry, which is also present in the Fourier transform. Therefore, the diffraction pattern would change if the tube is rotated around its axis.

The scattering potential $V(r)$ is calculated from the tabularized values of Gaussian fits to relativistic Hartree-Fock calculations given in [55]. This derivation can be found e.g. in [54]. Within the WPOA (and planar approximation of the Ewald sphere), the diffraction pattern is calculated from a Fourier transform of the projected potential. Alternatively, the phase shift in the exit plane wave function can be calculated from the projected potential (phase object approximation). The diffraction pattern is then calculated from a Fourier transform of the exit plane wave function.

Computing diffraction patterns by a fast Fourier transformation of the exit plane wave function is much faster than the path summation calculation shown in section III The fast Fourier transform typically takes less than a minute, while the real space summation needs several hours. However, it implies approximating a section of the Ewald sphere as a plane.

\section{Qualitative description of the diffraction pattern}

The diffraction pattern of a single-walled carbon nanotube can be well separated into features that depend on the nanotube diameter, and others which depend on the rolling angle of the graphene sheet. The most prominent feature is the equatorial line, which is similar to a double-slit interference pattern. The periodicity of the intensities on this line is related only to the nanotube diameter.

From further peaks in the pattern we can determine the orientation of the (reciprocal) graphene hexagonal lattices. A simulated diffraction pattern for a $(15,06)$ nanotube, and a part of the reciprocal graphene lattice, is shown in Figure 5 Two separate sets of peaks that correspond to the top and bottom graphene layer are visible for chiral nanotubes (which coincide for armchair and zigzag nanotubes). The peaks appear as streaks due to the curvature of the graphene sheet.

The orientation of the graphene lattice, i.e. the rolling angle of the nanotube, can be calculated independent of the incidence angle from the relative distances of the peaks from the equatorial line. This measure is also independent of the scale (camera length) or a diffraction astigmatism. Thus, the rolling angle can be reliably and precisely measured with a precision of up to $0.1^{\circ}$. Using e.g. the distances $d_{1}-d_{3}$ in Figure 5 the rolling vector is [22]:

$\alpha=\arctan \left(\frac{1}{\sqrt{3}} \cdot \frac{d_{2}-d_{1}}{d_{3}}\right)=\arctan \left(\frac{1}{\sqrt{3}} \cdot \frac{2 d_{2}-d_{3}}{d 3}\right)_{(4)}$

For identifying the indices of a nanotube from a diffraction pattern, a measurement of the diameter and angle from the pattern as described above can be used as a starting point. Then, the simulated images are needed. Once a matching structure is found, the most important aspect for an unambiguous identification is to exclude all other possible nanotube structures 


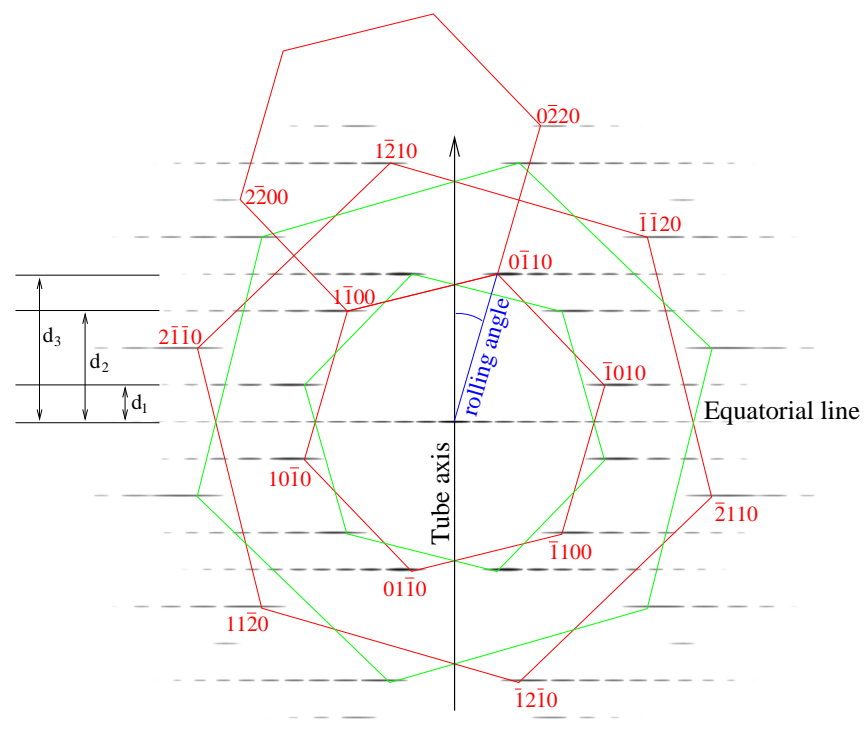

Figure 5: Orientation of the reciprocal graphene lattices in the SWNT diffraction pattern. Two sets of peaks that correspond to the top and bottom graphene layer in the nanotube are visible. The MillerBravais indices for one of the two hexagonal lattices are given. A rolling angle of $0^{\circ}$ corresponds to a zig-zag nanotube, $30^{\circ}$ represents an armchair tube. It is not recommended to measure the angle as indicated in the diagram, since the indicated angle is only true for an exactly normal incidence of the beam on the tube. Instead, the angle can be reliably calculated from the relative distances of the peaks to the equatorial line, indicated by $d_{1}, d_{2}$ and $d_{3}$. The streaked shape of the diffraction peaks is due to the curvature of the graphene sheet.

(within a reasonable range of angle and diameter). For a sufficiently sharp diffraction pattern, there is usually only one set of indices $(n, m)$ for which the simulated diffraction pattern matches the experimental data. Only for very large diameter nanotubes, the parameters (diameter and angle) of the different candidates are spaced so close together that they can not be distinguished.

\section{Accuracy of the simulation methods}

The single-walled carbon nanotube is one of the few systems well described by the weak phase object approximation (WPOA). In addition to calculations [3], the validity of the WPOA is demonstrated in an impressive way by the oversampling and iterative phase retrieval for a nanotube from diffraction intensities [7]. The reconstruction in [7] is based on the WPOA, and has worked for the large double-walled carbon nanotube. However the iterative phase retrieval is not possible from our diffraction data, because it requires the fully coherent illumination of a short nanotube section which is available only with field-emission electron sources.

From a Fourier transformation of the projected potential, one can quickly calculate a simulated diffraction pattern (e.g. Figure 5). This approach approximates the visible section of the Ewald sphere as a plane. The computationally more expensive path summation approach (section IIIA) naturally includes the curvature. The curvature effect is more pronounced at lower acceleration voltages. The difference can be neglected for normal incidence, but it is clearly seen in diffraction images for non-normal incidence. For non-normal incidence, the peak positions are no longer symmetric with respect to the origin. The actual difference between curved and planar approximation to the Ewald sphere depends on the tube structure and actual incidence angle. We find that for an incidence angle within $\approx 10^{\circ}$ of normal incidence, a safe assignment of nanotube indices using the planar approximation to the Ewald sphere is possible.

\section{DIFFRACTION IMAGES}

\section{A. Experimental procedure}

All SWNT diffraction patterns were obtained using a Zeiss $912 \Omega$ transmission electron microscope. It is equipped with a (thermal) $\mathrm{LaB}_{6}$ electron gun, a Köhler illumination system, and an energy filter. Images can be taken on two CCD cameras with different fields of views. The diffraction patterns are recorded on image plates. The image plates provide a very high sensitivity and dynamic range, which is not matched by any CCD camera. CCD cameras, and also conventional film, suffer from a "blooming" effect: Intensity from strongly excited, saturated pixels spreads out into the nearby regions, occulting weak intensity diffraction peaks.

Unless noted otherwise, the following procedure and conditions ("standard conditions") are used for obtaining diffraction patterns. The microscope is operated at an acceleration voltage of only $60 \mathrm{kV}$, clearly below the threshold for knock-on damage in carbon nanotubes which is $87 \mathrm{kV}$ [56]. The Köhler illumination condition is used with an illumination angle between 0.1 and $0.2 \mathrm{mrad}$. The illumination is limited to a straight section of the carbon nanotube using the condenser aperture. The smallest condenser aperture, which has a diameter of $5 \mu \mathrm{m}$, produces an illuminated region (demagnified image of the aperture) with a diameter of approximately $130 \mathrm{~nm}$. An image of a carbon nanotube illuminated under these conditions is shown in Figure 6 Before switching to diffraction mode, the focus is tuned to the minimum contrast condition. The energy filter is set to a width of $15-20 \mathrm{eV}$. The diffraction image is recorded on image plates with a camera length of $450 \mathrm{~mm}$ or $580 \mathrm{~mm}$ and exposure times of 4 or 5 minutes.

As a reference, diffraction images are recorded under the same conditions, except for a shorter exposure time, from the metal contacts. These contacts are polycrystalline, producing Debye-Scherrer ring type of diffraction patterns. From these, any diffraction astigmatism remaining after alignment can be detected and compensated, and a precise scale is obtained. An astigmatism of a few percent may already lead to an incorrect assignment of the nanotube indices. 


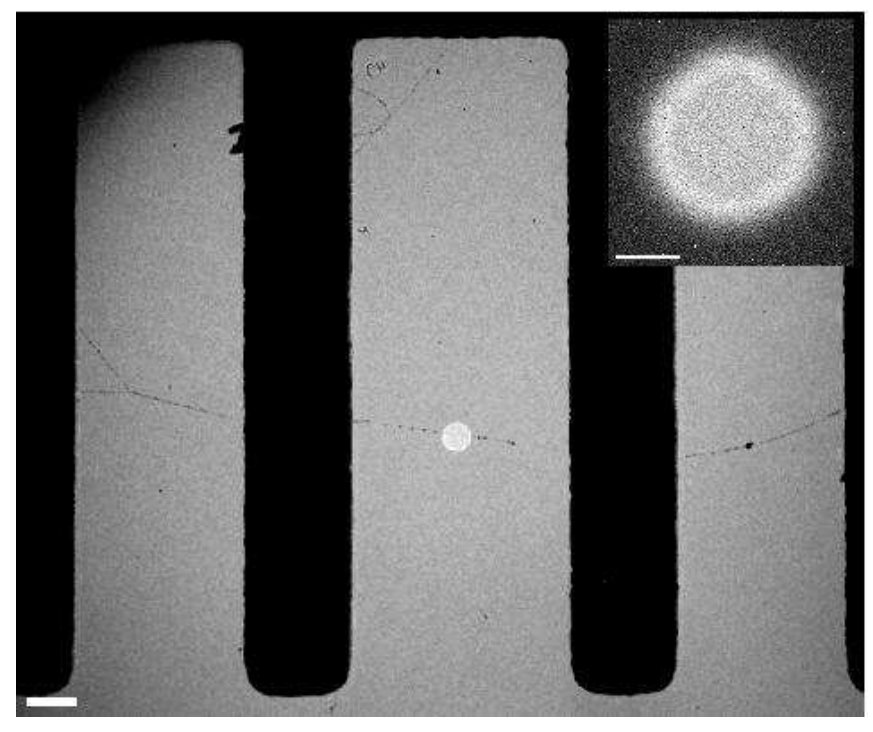

Figure 6: Inset: An individual SWNT within the illuminated region of the smallest condenser aperture. Main image: Overlay of a TEM image of the fully illuminated sample with an image of smallest condenser aperture (bright spot in the center). This overlay shows the illuminated section of the nanotube (for a diffraction pattern) in relation to the sample structure. The illuminated region for the smallest condenser aperture has a diameter of $130 \mathrm{~nm}$. Scale bar is $200 \mathrm{~nm}$, and $50 \mathrm{~nm}$ for the inset.

\section{B. Discussion of experimental parameters}

Previous diffraction work on individual single-walled carbon nanotubes [22] was performed in transmission electron microscopes equipped with a field-emission electron source, resulting in highly coherent illumination. Therefore, diffraction images could be recorded with short exposure times, probably before significant damage to the nanotubes occurred. However, it can not be excluded that surface reconstruction and dimensional changes due to the electron irradiation [57] change the nanotube structure during the exposure.

Using a thermal emitter, we need longer exposure times. Operation below the threshold for knock-on damage makes it possible to have stable conditions throughout the long exposure times. It is possible to obtain several diffraction images from the same nanotube section without loss of quality. Further, minimizing damage is important if transport or Raman investigations are to be carried out on the same nanotube.

Figure 7 shows three diffraction patterns obtained on different nanotubes. All diffraction images obtained in this way show intense radial lines starting from the central peak. These are due to electrons scattered into high angles at the condenser aperture. They are partially shadowed by the structure of the sample. A shadow of the sample structure is visible in the diffraction pattern due to this effect.

With a sufficiently sharp diffraction pattern, an unambiguous assignment of the nanotube structure, or nanotube indices $(n, m)$, is possible. A straight, clean nanotube is required - a curved nanotube is not a $1 \mathrm{D}$ periodic structure. In the highresolution images of suspended nanotubes we are able to ob-
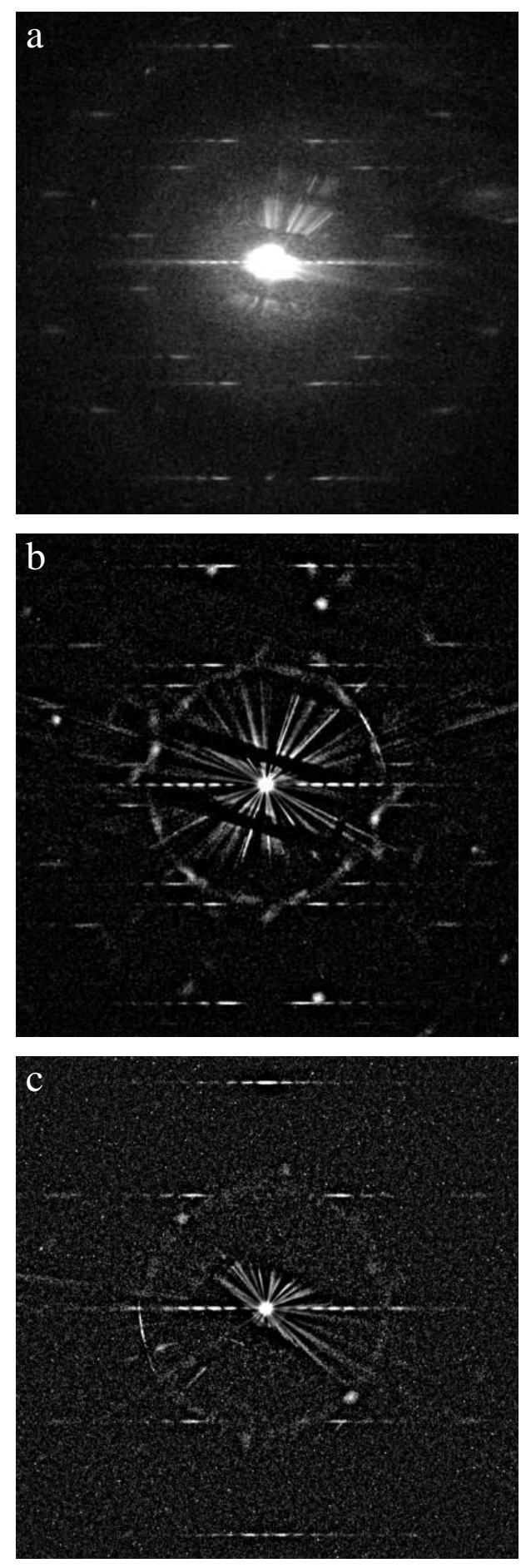

Figure 7: Examples of diffraction patterns. (a) $(24,11)$ nanotube, (b) $(16,09)$ nanotube, (c) $(13,13)$ "armchair" nanotube. A background subtraction was done on images (b) and (c).

tain a sharp image only from the ends, due to vibrations of the central part. Such vibrations are not a problem for diffraction analysis: A translation of the nanotube does not change the diffraction pattern. Only if the vibrations are so large that the orientation of the tube varies, it leads to a blurred diffraction 
image.

Although all diffraction images were recorded at the same microscope, it seems that the key ingredients for a singletube diffraction analysis are the small condenser aperture, image plates for recording of the diffraction patterns, and very straight nanotubes. The condenser aperture limits the illumination to the region of interest (the nanotube section). Selecting the area downstream of the sample (using only the selected area aperture) fails, since many other contributions (e.g. reflections from the metal contact, inelastic contributions) still reach the detector. The smallest unwanted contributions will occult the very weak diffraction intensities from the small number of atoms in our nanotube. We have seen that the energy filter is not necessary, but improves the image quality. The Köhler illumination condition provides a homogeneous incident beam; however, convergent-beam electron diffraction (shown below) is also possible on individual nanotubes.

\section{Index assignment}

For the analysis, the diffraction images are rotated to have the equatorial line in a horizontal direction. If any astigmatism is found in the reference patterns, it is compensated by rescaling and shearing the images by the appropriate amount. In some cases a background subtraction is useful. The above steps can be done with the ImageJ software and The Gimp (there is a version capable of manipulating 16 and 32-bit images, called FilmGimp).

The rotated, background subtracted and rescaled images are then imported in xfig. Here, the features in the image are marked (Figure 8). Then, simulated images are placed instead of the experimental underneath the marks, to find those which match. We find that for a sharp diffraction pattern there is exactly one set of indices $(n, m)$ for which the simulated image matches the experimental one. The indices and the incidence angle of the simulated pattern are varied to find the matching set of parameters. Thus, both the nanotube structure and the incidence angle are measured from the diffraction pattern. Once a matching pattern is found, we make sure that all nearby indices do not match, independent of the incidence angle. By excluding all except one pair of indices $(n, m)$ we obtain an unambiguous assignment.

The smallest individual carbon nanotube that was identified is a $(7,7)$ nanotube, which has a diameter of $0.94 \mathrm{~nm}$. One of the largest identified nanotubes is $(39,26)$ which has a diameter of $4.44 \mathrm{~nm}$.

Figure 9 shows the statistics of indices obtained from nanotubes all grown in the same CVD process. We use 4-5 nm $\mathrm{Ni}$ nanoparticles as catalyst, and methane as carbon feedstock with flows of $1.2 \mathrm{~L} / \mathrm{min}$ of methane and $0.6 \mathrm{~L} / \mathrm{min}$ of $\mathrm{H}_{2}$. The synthesis temperature is $900^{\circ} \mathrm{C}$ and the duration $10 \mathrm{~min}$. We describe the CVD process in detail in [42].
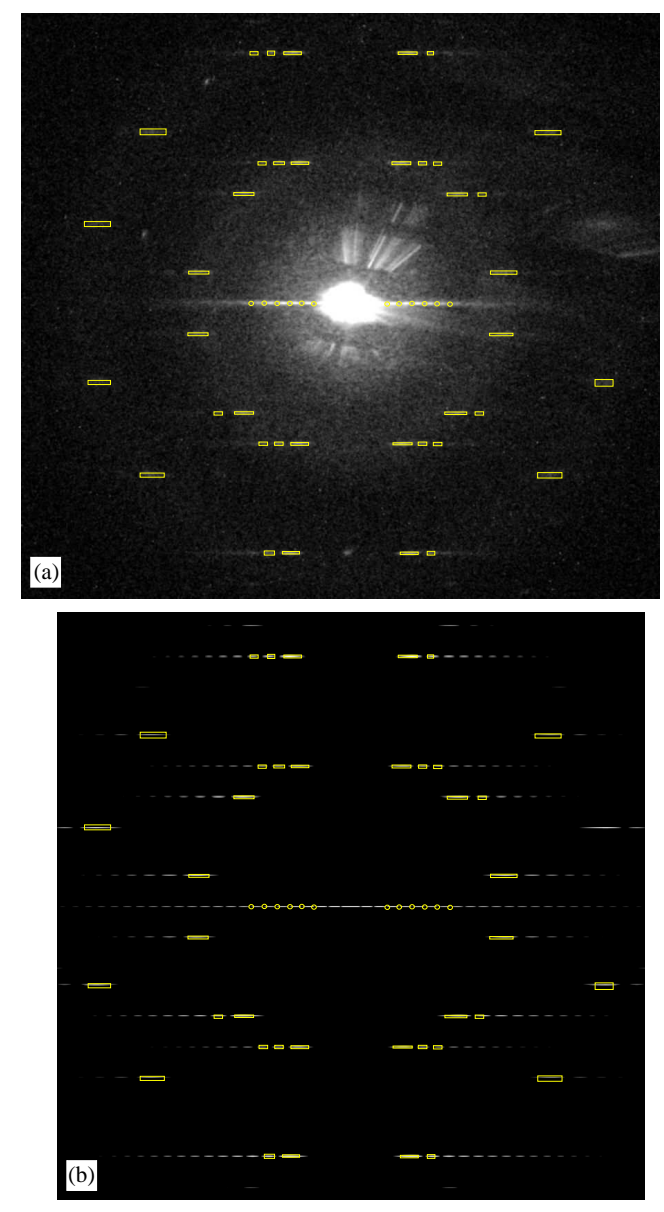

Figure 8: Index assignment: (a) The peaks in the experimental diffraction pattern (from figure [7 ) are marked. A box is drawn around the streaked graphene peaks, and in the equatorial line the minima are marked. In (b), the experimental image is replaced by a simulated one, without change in the marks. The indices and incidence angle in the simulation are changed until the pattern matches. Here, the pattern matches only one pair of indices $(n, m)=(24,11)$. The incidence angle is within $3^{\circ}$ of normal incidence.

\section{Discussion of the index distribution}

Figure 9 clearly shows that the rolling angle is not randomly distributed, but is grouped towards the armchair orientation in this material. This is a direct measurement and it includes metallic nanotubes. Fluorescence spectroscopic results [35, 36] show a similar trend, but are limited to semiconducting tubes and biased by a structure-dependent fluorescence quantum yield. The Raman study in Ref. [32] finds a more or less random distribution of nanotube indices in HiPCO nanotubes. Note that all these index distributions are obtained from nanotubes grown with different methods and parameters. As demonstrated in [36] the index distribution strongly depends on growth conditions. We can compare the angular selectivity in our material with fluorescence studies of nanotubes produced by alcohol catalytic CVD (ACCVD) under optimized conditions [36] and of nanotubes grown from solid-supported $\mathrm{Mo} / \mathrm{Co}$ catalyst (MoCo) [35], under the as- 


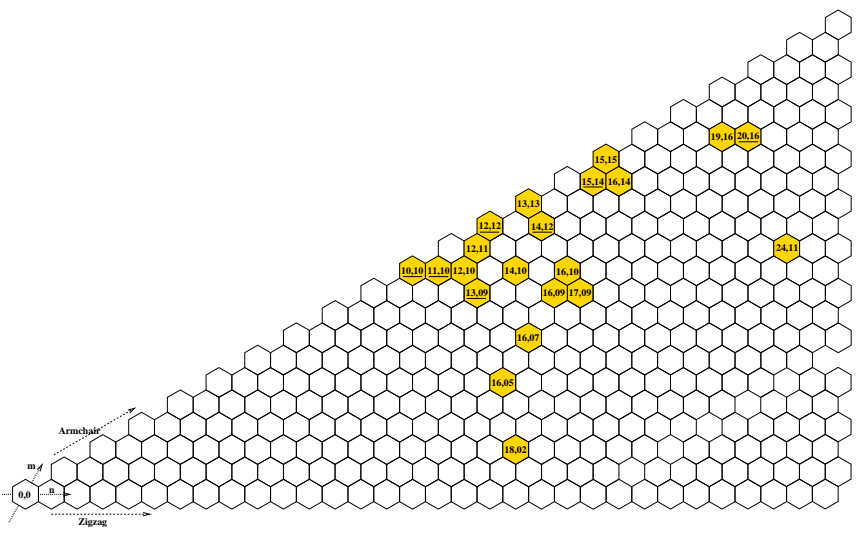

Figure 9: Nanotube indices of 28 tubes grown in the same CVD process. The underlined indices were encountered twice. The rolling angle is not randomly distributed, but is close to the armchair direction $\left(30^{\circ}\right)$ in the majority of the nanotubes in this material.

sumption of a structure-independent fluorescence yield. An angle of $30^{\circ}$ represents armchair nanotubes, $0^{\circ}$ a zigzag tube, and an average of $15^{\circ}$ is expected for a random distribution. The average angle in the MoCo material, calculated from the fractional intensities listed in [35], is $22.8^{\circ}$. The optimized ACCVD appears to yield predominantly $(6,5)\left(27^{\circ}\right)$ and $(7,5)$ $\left(24.5^{\circ}\right)$, but also significant amounts of $(8,3)\left(15,3^{\circ}\right)$ and $(8,4)$ $\left(19.1^{\circ}\right)$. The intensities in [36] are given only in a graphical representation, from which we estimate an average angle of $\approx 23^{\circ}$. In our sample the mean angle is $25^{\circ}$ if we include all nanotubes, and $24^{\circ}$ if we exclude the metallic tubes for a better comparison with the fluorescence spectroscopic results. Due to the much larger diameters obtained in our CVD process, there are many more different nanotube species possible within a given angle interval. The angular selectivity towards armchair tubes, however, is at least similar to the samples in [35] or [36].

\section{E. Convergent-beam electron diffraction}

Although nanoarea electron diffraction using the Köhler illumination condition with a small condenser aperture has proven to be very reliable, we would like to show also the possibility of convergent-beam electron diffraction (CBED) on individual single-walled carbon nanotubes. Here, the illuminating electron beam converges to form a small probe of a few nm on a straight nanotube section. Consequently, the diffraction pattern consists of extended discs instead of sharp points. It is possible to investigate even smaller nanotube sections in this way. An unambiguous nanotube index assignment, however, is not possible (it could be possible in combination with a very accurate diameter estimate from a high-resolution image, which is not available in our case). The image is again recorded on image plates with an exposure time of five minutes.

A convergent beam electron diffraction pattern of an individual single walled nanotube is shown in Figure 10 A dis-
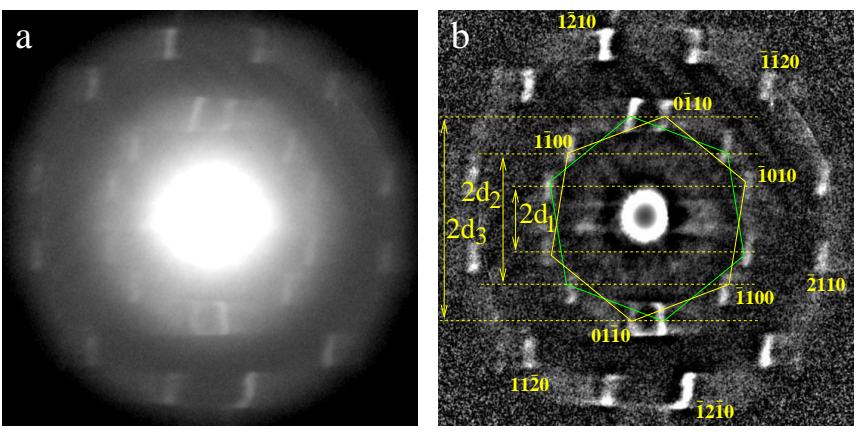

Figure 10: Convergent beam electron diffraction (CBED) image of an individual single-walled carbon nanotube. Original (a) and background subtracted image (b). The orientation of the graphene lattice is visible, but the equatorial line is not. The illuminated nanotube section is visible in each of the diffraction spots. In (b) part of the reciprocal graphene lattice and indices according to Fig. 5] are shown. The hexagons do not precisely match the peaks due to non-normal incidence. The rolling angle of the nanotube can be calculated from the distances $\mathrm{d}_{1}-\mathrm{d}_{3}$ according to equation (4), and in this example we obtain $12.5 \pm 0.5^{\circ}$

torted image of the tube is visible in each spot (in fact the tube is straight). In this way the rolling angle of a short segment can be detected. Unfortunately, the periodicity in the equatorial line, which provides the diameter, can not be measured. Even if the indices can not be determined, CBED may be useful for an analysis of the rolling angles of carbon nanotubes. The angle can be measured from the relative distances as indicated in Fig. 10

\section{F. Diffraction on related nanotube structures}

Diffraction is possible on a wide range of nanotube structures. Using the same experimental conditions, we have obtained diffraction patterns also from double-walled nanotubes (DWNTs), multi-walled nanotubes and small bundles (not shown). In double-walled nanotubes, multi-walled nanotubes, and nanotube bundles, multiple orientations of the graphene lattice are visible in a diffraction pattern. Considerations for assigning the indices of DWNTs can be found in [5]. An index assignment for bundles and MWNTs is often not unique, as it is not clear which tube diameter belongs to a specific orientation.

\section{CONCLUSIONS AND OUTLOOK}

A diffraction analysis of carbon nanotubes is presented, including a novel sample preparation method, description of simulation methods, and electron diffraction at an energy below the threshold for knock-on damage. Experimental parameters are described and discussed, permitting the reader to perform a similar analysis (e.g. statistics of tube indices). We have shown single-nanotube diffraction in the Köhler illumination condition as well as convergent-beam electron diffrac- 
tion. The goal of this study is a reliable determination of the structural indices for a suspended nanotube in our freestanding structures. This is achieved for the majority of the candidate nanotubes.

The importance of a reliable, non-destructive analysis of the nanotube indices lies in combining the structural analysis with other experiments on the same object. This becomes possible through our lithographically defined free-standing structure. We are currently collecting data from single-tube transport measurements and Raman spectroscopy using similar sample structures as shown here [45, 58]. These are, finally, measurements on well-defined molecules that allow a correlation of electronic and vibrational properties with structural information. We point out that the free-standing structure can be designed almost arbitrarily by lithography so that complex free- standing structures, including nanotubes, can be prepared for a variety of novel experiments.

\section{Acknowledgments}

The authors acknowledge financial support by the BMBF project INKONAMI (Contract No. 13N8 402) and the EU project CANAPE. We thank xlith.com for lithography services. We thank Prof. M. Ruehle and his group for support with TEM, especially C. Koch for very helpful discussions. M. P. acknowledges support from Dr. B. Chaudret's group, Dr. V. Jourdain, Dr. P. Poncharal and Prof A. Zahab.
[1] X. F. Zhang, X. B. Zhang, G. Van Tendeloo, S. Amelinckx, M. Op de Beeck, and J. Van Landuyt. Carbon nano-tubes; their formation process and observation by electron microscopy. $J$. Cryst. Growth, 130:368, 1993.

[2] X. B. Zhang, X. F. Zhang, S. Amelinckx, G. Van Tendeloo, and J. Van Landuyt. The reciprocal space of carbon nanotubes: a detailed interpretation of the electron diffraction effects. Ultramicr., 54:237, 1994.

[3] A. A. Lucas, V. Bruyninckx, Ph. Lambin, D. Bernaerts, S. Amelinckx, J. Van Landuyt, and G. Van Tendeloo. Electron diffraction by carbon nanotubes. Scanning Microscopy, 12:415, 1997.

[4] S. Amelinckx, A. Lucas, and P. Lambin. Electron diffraction and microscopy of nanotubes. Rep. Prog. Phys., 62:1471, 1999.

[5] M. Kociak, K. Hirahara, K. Suenaga, and S. Iijima. How accurate can the determination of chiral indices from of carbon nanotubes be? Eur. Phys. J. B., 32:457, 2003.

[6] M. Kociak, K. Suenaga, K. Hirahara, Y. Saito, T. Nakahira, and S. Iijima. Linking chiral indices and transport properties of double-walled carbon nanotubes. Phys. Rev. Lett, 89:155501, 2002.

[7] J. M. Zuo, I. Vartanyants, M. Gao, R. Zhang, and L. A. Nagahara. Atomic resolution imaging of a carbon nanotube from diffraction intensities. Science, 300:1419, 2003.

[8] K. Hirahara, S. Bandow, H. Kataura, M. Kociak, and S. Iijima. Stretching of carbon-carbon bonds in a $0.7 \mathrm{~nm}$ diameter carbon nanotube studied by electron diffraction. Phys. Rev. B, 70:205422, 2004.

[9] J. M. Cowley and F. A. Sundell. Nanodiffraction and dark-field stem characterization of single-walled carbon nanotube ropes. Ultramicr., 68:1, 1997.

[10] L.-C. Qin, S. Iijima, H. Kataura, Y. Maniwa, S. Suzuki, and Y. Achiba. Helicity and packing of single-walled carbon nanotubes studied by electron nanodiffraction. Chem. Phys. Lett., page 101, 1997.

[11] J. M. Cowley, P. Nikolaev, A. Thess, and R. E. Smalley. Electron nano-diffraction study of carbon single-walled nanotube ropes. Chem. Phys. Lett., 265:379, 1997.

[12] Ph. Lambin and A. A. Lucas. Quantitative theory of diffraction by carbon nanotubes. Phys. Rev. B, 56:3571, 1997.

[13] D. Bernaerts, A. Zettl, N. G. Chopra, A. Thess, and R. E. Smalley. Electron diffraction study of single-wall carbon nanotubes. Solid State Commun., 105:145, 1997.
[14] R. R. He, H. Z. Jin, J. Zhu, Y. J. Yan, and X. H. Chen. Physical and electronic structure in carbon nanotubes. Chem. Phys. Lett., 298:170, 1998.

[15] H.-Z. Jin, R.-H. He, and J. Zhu. Helicity and inter-tube bonding in bundles of single-walled carbon nanotubes. J. Electron Microsc., 48:339, 1999.

[16] L. Henrard, A. Loiseau, C. Journet, and P. Bernier. Study of the symmetry of single-wall nanotubes by electron diffraction. Eur. Phys. J. B, 13:661, 2000.

[17] J.-F. Colomer, L. Henrard, Ph. Lambin, and G. Van Tendeloo. Electron diffraction study of small bundles of single-wall carbon nanotubes with unique helicity. Phys. Rev. B, page 125425 , 2001.

[18] J.-F. Colomer, L. Henrard, Ph. Lambin, and G. Van Tendeloo. Electron diffraction and microscopy of single-wall carbon nanotube bundles produced by different methods. Eur. Phys. J. $B, 27: 111,2002$.

[19] J.-F. Colomer, L. Henrard, P. Launois, G. Van Tendeloo, A. A. Lucas, and $\mathrm{Ph}$. Lambin. Interpretation of electron diffraction from carbon nanotube bundles presenting precise helicity. Phys. Rev. B, 70:075408, 2004.

[20] Sumio Iijima and Toshinari Ichihashi. Single-shell carbon nanotubes of 1-nm diameter. Nature, 363:603-605, 1993.

[21] L. C. Qin, T. Ichihashi, and S. Iijima. On the measurement of helicity of carbon nanotubes. Ultramicr., 67:181, 1997.

[22] M. Gao, J. M. Zuo, R. D. Twesten, I. Petrov, L. A. Nagahara, and R. Zhang. Structure determination of individual single-wall carbon nanotubes by nanoarea electron diffraction. Appl. Phys. Lett., 82:2703, 2003.

[23] Jannik C. Meyer, Dirk Obergfell, Shihe Yang, Shangfeng Yang, and Siegmar Roth. Transmission electron microscopy and transistor characteristics of the same carbon nanotube. Appl. Phys. Lett., 85:2911, 2004.

[24] A. A. Lucas, V. Bruyninckx, and Ph. Lambin. Calculating the diffraction of electrons or x-rays by carbon nanotubes. Europhys. Lett., 35:355, 1996.

[25] L.-C. Qin. Electron diffraction from cylindrical nanotubes. $J$. Mater. Res., 9:2450, 1994.

[26] L.-C. Qin. Measuring the true helicity of carbon nanotubes. Chem. Phys. Lett., 297:23, 1998.

[27] Z. Liu and L.-C. Qin. A direct method to determine the chiral indices of carbon nanotues. Chem. Phys. Lett., 408:75, 2005.

[28] Z. Liu, Q. Zhang, and L.-C. Qin. Accurate determination 
of atomic structure of multiwalled carbon nanotubes by nondestructive nanobeam electron diffraction. Appl. Phys. Lett., 86:191903, 2005.

[29] A. Hashimoto, K. Suenaga, A. Gloter, K. Urita, and S. Iijima. Direct evidence for atomic defects in graphene layers. Nature, 430:870, 2004.

[30] A. Hashimoto, K. Suenaga, K. Urita, T. Shimada, T. Sugai, S. Bandow, H. Shinohara, and S. Iijima. Atomic correlation between adjacent graphene layers in double-wall carbon nanotubes. Phys. Rev. Lett., 94:045504, 2005.

[31] R. R. Meyer, S. Friedrichs, A. I. Kirkland, J. Sloan, J. L. Hutchinson, and M. L. H. Green. A composite method for the determination of the chirality of single-walled carbon nanotubes. J. Microsc., 212:152, 2003.

[32] H. Telg, J. Maultzsch, S. Reich, F. Hennrich, and C. Thomsen. Chirality distribution and transition energies of carbon nanotubes. Phys. Rev. Lett., 93:177401, 2004.

[33] A. Jorio, R. Saito, J. H. Hafner, C. M. Lieber, M. Hunter, T. McClure, G. Dresselhaus, and M. S. Dresselhaus. Structural (n,m) determination of isolated single-wall carbon nanotubes by resonant raman scattering. Phys. Rev. Lett., 86:1118, 2001.

[34] S. M. Bachilo, M. S. Strano, C. Kittrell, R. H. Hauge, R. E. Smalley, and R. B. Weisman. Structure-assigned optical spectra of single-walled carbon nanotubes. Science, 298:2361, 2002.

[35] S. M. Bachilo, L. Balzano, J. E. Herrera, F. Pompeo, D. E. Resasco, and R. B. Weisman. Narrow (n,m)-distribution of singlewalled carbon nanotubes grown using a solid supported catalyst. J. Am. Chem. Soc., 125:11186, 2003.

[36] Y. Miyauchi, S. Chiashi, Y. Murakami, Y. Hayashida, and S. Maruyama. Fluorescence spectroscopy of single-walled carbon nanotubes synthesized from alcohol. Chem. Phys. Lett., 387:198, 2004.

[37] J. W. G. Wildoer, L. C. Venema, A. G. Rinzler, R. E. Smalley, and C. Dekker. Electronic structure of atomically resolved carbon nanotubes. Nature, 391:59, 1998.

[38] T. W. Odom, J. L. Huang, P. Kim, and C. M. Lieber. Atomic structure and electronic properties of single-walled carbon nanotubes. Nature, 391:62, 1998.

[39] L. C. Venema, V. Meunier, P. Lambin, and C. Dekker. Atomic structure of carbon nanotubes from scanning tunneling microscopy. Phys. Rev. B, 61:2991, 2000.

[40] L. Vitali, M. Burghard, M. A. Schneider, L. Liu, S. Y. Wu, C. S. Jayanthi, and K. Kern. Phonon spectromicroscopy of carbon nanostructures with atomic resolution. Phys. Rev. Lett., 93:136103, 2004.

[41] M. Machon, S. Reich, H. Telg, J. Maultzsch, P. Ordejon, and C. Thomsen. Strength of radial breathing mode in single-walled carbon nanotubes. Phys. Rev. B, 71:035416, 2005.

[42] M. Paillet, V. Jourdain, P. Poncharal, J.-L. Sauvajol, A. Zahab, J. C. Meyer, S. Roth, N. Cordente, C. Amiens, and B. Chau- dret. Versatile synthesis of individual single-walled carbon nanotubes from nickel nanoparticles for the study of their physical properties. J. Phys. Chem. B, 108:17112-17118, 2004.

[43] M. Paillet, V. Jourdain, P. Poncharal, J.-L. Sauvajol, A. Zahab, J. C. Meyer, S. Roth, N. Cordente, C. Amiens, and B. Chaudret. Growth and physical properties of individual single-walled carbon nanotubes. Diamond and Related Materials, 14:1426, 2005.

[44] R. Seidel, G. S. Duesberg, E. Unger, A. P. Graham, M. Liebau, and F. Kreupl. Chemical vapor deposition growth of singlewalled carbon nanotubes at $600^{\circ} \mathrm{c}$ and simple growth model. $J$. Phys. Chem. B, 108:1888, 2004.

[45] J. C. Meyer, M. Paillet, T. Michel, A. Moreac, A. Neumann, G. S. Duesberg, S. Roth, and J. L. Sauvajol. Raman modes of index-identified single-walled carbon nanotubes. To be published. http://arxiv.org/cond-mat/0506666.

[46] R. Gevers and M. David. Relativistic theory of electron and position diffraction at high and low energy. Phys. Stat. Sol., 113:665, 1962.

[47] C. J. Humphreys. The scattering of fast electrons by crystals. Rep. Prog. Phys. 42, 42:1825, 1979.

[48] J. C. H. Spence. High-Resolution Electron Microscopy. Oxford University Press, 2003.

[49] R. P. Feynman. Space-time approach to non-relativistic quantum mechanics. Rev. Mod. Phys., 20:367, 1948.

[50] R. P. Feynman and A. R. Hibbs. Quantum Mechanics and Path Integrals. McGraw-Hill, New York, 1965.

[51] E. J. Kirkland. Advanced Computing in Electron Microscopy. Plenum Press, New York, 1998.

[52] P. R. Buseck, J. M. Cowley, and L. Eyring. High-Resolution Transmission Electron Microscopy. Oxford University Press, 1988.

[53] C. J. Humphreys. The scattering of fast electrons by crystals. Rep. Prog. Phys., 42:122, 1979.

[54] L.-M. Peng. Electron atomic scattering factors and scattering potentials of crystals. micron, 30:625, 1999.

[55] P. A. Doyle and P. S. Turner. Relativistic hartree-fock x-ray and electron scattering factors. Acta Cryst. A, 24:390, 1968.

[56] B. W. Smith and E. Luzzi. Electron irradiation effects in single wall carbon nanotubes. J. Appl. Phys., 90:3509, 2001.

[57] P. M. Ajayan, V. Ravikumar, and J.-C. Charlier. Surface reconstruction and dimensional changes in single-walled carbon nanotubes. Phys. Rev. Lett., 81:1437, 1998.

[58] J. C. Meyer, M. Paillet, J.-L. Sauvajol, D. Obergfell, A. Neumann, G.S. Duesberg, and S. Roth. Novel freestanding nanotube devices for combining tem and electron diffraction with raman and transport. In Electronic Properties of Novel Materials, New York, 2005. American Institute of Physics. In press. 